Pacific Journal of Mathematics

COMPARISON OF DE RAM AND DOLBEAULT
COHOMOLOGY FOR PROPER SURJECTIVE MAPPINGS 


\section{COMPARISON OF DE RHAM AND DOLBEAULT COHOMOLOGY FOR PROPER SURJECTIVE MAPPINGS}

R. O. WELLS, JR.

In this paper it is shown that if $\pi: \widetilde{X} \rightarrow X$ is a proper holomorphic surjection of equidimensional complex manifolds then the induced mapping $\pi^{*}: H^{q}\left(X, \Omega_{X}^{p}\right) \rightarrow H^{q}\left(\widetilde{X}, \Omega_{\widetilde{X}}^{p}\right)$ on Dolbeault groups is injective. As a consequence one obtains the inequality $h^{p, q}(X) \leqq h^{p, q}(\tilde{X})$ for the Hodge numbers of $X$ and $\tilde{X}$. This result is valid also in the case of vector bundle coefficients, and can be generalized to the case of nondiscrete fibres of the mapping $\pi$ (non equidimensional case) by the imposition of a Kählerian condition on $\tilde{X}$. Corresponding results for differentiable mappings are formulated and proved. Illustrative examples are provided to show the necessity of the various assumptions made.

1. Introduction. Let $\pi: \widetilde{X} \rightarrow X$ be a surjective proper holomorphic mapping of complex manifolds ${ }^{1}$. Our main result in this paper (Theorem 4.1) asserts that if $\tilde{X}$ is a Kähler manifold, then the mapping $\pi$ induces injections

$$
\begin{aligned}
\pi^{*}: H^{q}\left(X, \Omega_{X}^{p}\right) & \longrightarrow H^{q}\left(\widetilde{X}, \Omega_{\widetilde{X}}^{p}\right) \\
\pi^{*}: H^{r}(X, C) & \longrightarrow H^{r}(\widetilde{X}, C)
\end{aligned}
$$

on the Dolbeault and de Rham groups, respectively. A consequence of this is that we have inequalities

$$
\begin{aligned}
b_{j}(\widetilde{X}) & \geqq b_{j}(X) \\
h^{p, q}(\widetilde{X}) & \geqq h^{p, q}(X)
\end{aligned}
$$

for the Betti numbers and Hodge numbers respectively (in the case that $\tilde{X}$ and $X$ are compact, for instance). If $\pi: \widetilde{Y} \rightarrow Y$ is a proper surjective differentiable mapping of even dimensional orientable manifolds and $\widetilde{Y}$ is a symplectic manifold, then there is a natural generalization of the notion of the "degree of $\pi$ ". Under the hypothesis that this degree is not zero,

$$
\pi^{*}: H^{r}(Y, R) \longrightarrow H^{r}(\widetilde{Y}, R),
$$

is an injection (Theorem 4.4) (cf. also Borel-Haefliger [2]).

In the case that $X$ and $\tilde{X}$ above have the same dimension, then the conclusion (1.1) and (1.2) still holds without any Kähler assumption,

1 All manifolds considered in this paper are assumed to be paracompact. 
i.e., for arbitrary complex manifolds (Theorem 3.1). If $Y$ and $\tilde{Y}$ above have the same dimension, then the conclusions (1.3) hold without the symplectic assumption, but it is still necessary that $\operatorname{deg} \pi \neq 0$ (Theorem 3.2). This particular result is due to Hopf [10] (for compact manifolds), and we give a new proof of his result in this paper. Hopf showed that the induced mapping on integral homology was surjective modulo torsion. Dualizing gives the assertion that the induced map on cohomology injects. Hopf used the technique of Lefschetz including the ring structure on homology induced by intersection theory (this has been generalized in Borel-Haefliger [2]). Our techniques involve differential forms and currents, currents being de Rham's generalization of the singular chains and cycles used by Hopf. In particular the induced mapping on currents go in the same direction as the induced mapping on cycles, a fact we use very strongly in the proofs.

Grauert and Riemenschneider [8] proved that if $\pi: \widetilde{X} \rightarrow X$ is a proper modification of compact Kähler manifolds, then the induced mapping on Dolbeault groups is an injection, a special case of Theorem 3.1, mentioned in the previous paragraph. Their proof used Hopf's theorem along with the Hodge decomposition theorem for Kähler manifolds. Our proof is more direct and does not use any Kähler structure. Deligne [4] has the algebraic analogue of Grauert-Riemenschneider theorem for proper birational morphisms of smooth schemes over a field $k$. Aeppli [1] has also studied the problem of comparison of cohomology for proper modifications, and some of his results were generalized by Grauert and Riemenschneider to the case of complex spaces with singularities, which we do not consider in this paper.

The method of proof of our results is based on using resolutions of the sheaf $C$ or $\Omega_{X}^{p}$ by differential forms with $C^{\infty}$ and distribution coefficients (currents). This is similar in spirit to Serre's proof of his duality theorem (Serre [16]).

The theorems in this paper grew out of an investigation of the behavior of harmonic forms under proper modification, and it is in this context we hope to make applications of the injection theorems at a later date.

Section 2 is devoted to a discussion of the global behavior of differential forms and currents under proper surjections and the interaction of these concepts. In $\S 3$ we formulate and prove our results on comparison of cohomology for finitely sheeted ramified covering mappings, using some of the results from $\S 2$. In $\S 4$ the ideas of $\S 2$ and $\S 3$ are generalized to surjection with nondiscrete fibres. In this context the existence of a Kähler form plays a crucial role.

I would like to thank Reese Harvey, John Hempel, John Polking, 
and Oswald Riemenschneider for their useful comments in various discussions concerning this work.

2. Differential forms and currents. Let $X$ be a complex manifold of complex dimension $n$, let $\mathscr{E}^{p, q}(X)$ be the $\left(C^{\infty}\right)$ differential forms on $X$ of type $(p, q)$, and let $\mathscr{D}^{p, q}(X)$ be the compactly supported differential forms in $\mathscr{E}^{p, q}(X)$, equipped with their usual topologies. We define the vector space $\mathscr{K}^{p, q}(X)$ of currents of type $(p, q)$ on $X$ as the dual space of the topological vector space $\mathscr{D}^{n-p, n-q}(X)$ (cf. de Rham [5]). Moreover, if $E \rightarrow X$ is a holomorphic vector bundle on $X$, then we let $\mathscr{E}^{p, q}(X, E)$ be the $E$-valued differential forms on $X$ of type $(p, q)$ and by $\mathscr{D}^{p, q}(X, E)$, the $E$-valued forms of compact support as above. We let $\mathscr{K}^{p, q}(X, E)$ denote $E$-valued currents on $X$ of type $(p, q)$, defined to be the dual of the topological vector space $\mathscr{D}^{n-p, n-q}(X$, $E^{*}$ ) equipped with its natural topology where $E^{*}$ is the dual bundle to $E$ (cf. e.g., Serre [16]). More generally, we let $\mathscr{E}^{r}(Y)$ be the realvalued differential forms of total degree $r$ on an orientable differentiable manifold of real dimension $m$, and by $\mathscr{K}^{r}(Y)$ the (real) currents of degree $r$ on $X$ defined as the dual of $\mathscr{D}^{m-r}(Y)$, where once again $\mathscr{D}^{m-r}(Y)$ denotes the compactly supported differential forms of degree $m-r$ on $Y$. In general we will denote the duality pairing of a current $T \in \mathscr{K}^{r}(Y)$ with an element $\varphi \in \mathscr{D}^{m-r}(Y)$ by the notation $\langle T, \varphi\rangle$ (cf. de Rham [5]).

We now consider a proper surjective holomorphic mapping $\pi: \widetilde{X} \rightarrow$ $X$ where $X$ and $\tilde{X}$ are complex manifolds of the same complex dimension $n$. Then we have a homomorphism of complexes induced by $\pi$.
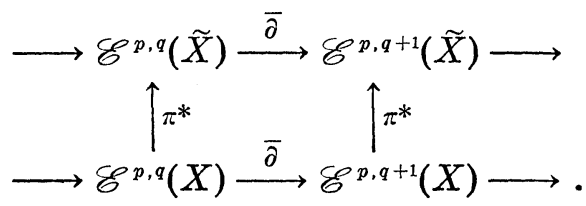

Similarly we have a homomorphism of complexes

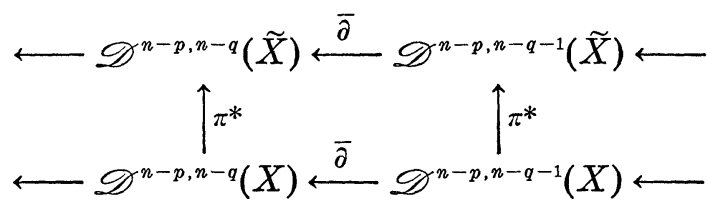

which induces by duality a homomorphism $\pi_{*}$ of the dual complexes

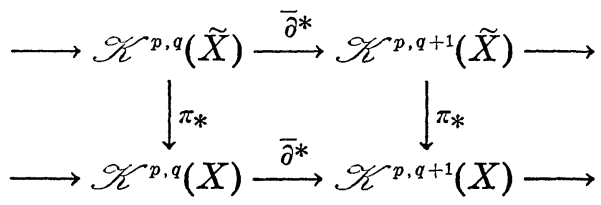


where $\bar{\partial}^{*}$ is the dual to $\bar{\partial}$ in the duality $\mathscr{K}^{p, q}(X)=\mathscr{D}^{p, q}(X)^{\prime}$. Here we use strongly the fact that $\pi$ is proper so that the support of the pullback of a form with compact support is still compact. We observe that $\bar{\partial}^{*}= \pm \bar{\partial}$, depending on the degree of the forms being acted on (integration by parts, cf. Serre [16]). Thus if we replace $\bar{\partial}^{*}$ by $\bar{\partial}$ in (2.3) we have

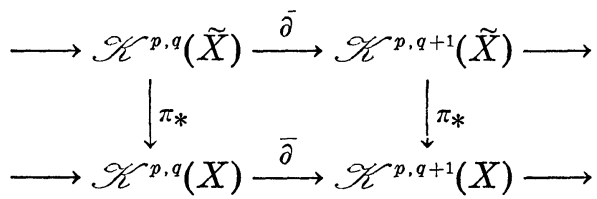

where each square is either commutative or anticommutative. Now consider the diagram

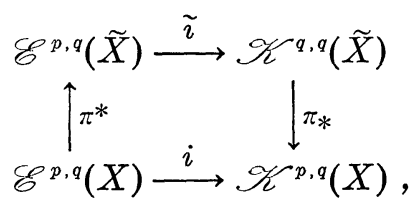

where $i$ and $\tilde{i}$ are the natural injections.

We want to study the obstruction to commutativity of the diagram (2.5). To do this we introduce a geometric invariant of the mapping $\pi$, the degree of $\pi$. We want to do this in general for differentiable manifolds. Suppose $\pi: \widetilde{Y} \rightarrow Y$ is a proper surjective differentiable mapping of orientable differentiable manifolds of the same real dimension $m$, then $\pi$ induces a mapping

$$
\mathscr{K}^{0}(\tilde{Y}) \stackrel{\pi_{*}}{\longrightarrow} \mathscr{K}^{0}(Y),
$$

induced by duality from

$$
\mathscr{E}^{m}(Y) \stackrel{\pi^{*}}{\longrightarrow} \mathscr{E}^{m}(\tilde{Y})
$$

Then we define $\mu=\pi_{*}(1)$, where the constant 1 is considered as a current on $\tilde{Y}$. Then since $d \pi_{*}(1)=\pi_{*}(d(1))=0$, it follows from the regularity theorem for currents that $\pi_{*}(1)$ is a function on $Y$ which is constant on each component of $Y$. We call $\mu$ the degree of the mapping $\pi$. Moreover, if $\pi$ has maximal rank on $\tilde{Y}$, and $y_{0} \in Y$, then

$$
\mu=\sum_{y \in \pi^{-1}\left(y_{0}\right)} \operatorname{sgn}(\operatorname{det}(d \pi)(y))
$$

where we mean by $\operatorname{det}(d \pi)(y)$ the determinant of the Jacobian matrix $d \pi$ at $y$ expressed in terms of oriented local coordinates at both $y$ and $y_{0}$. In other words, 


$$
d \pi: T_{y}(\tilde{Y}) \longrightarrow T_{y_{0}}(Y)
$$

is either orientation preserving or not and $\mu$ is the algebraic sum of the number of points in $\pi^{-1}(y)$ counting a point positively or negatively depending on the preservation of orientation or not. This result is proved in Federer [6], and is a special case of our results in $\$ 4$ where we generalize the notion of degree for symplectic manifolds. If $\pi$ is orientation preserving (as in the case of a complexanalytic map, for instance) then $\mu$ is the number of points in $\pi^{-1}\left(y_{0}\right)$, for any $y_{0} \in Y$.

We now have the following basic lemma concerning the commutativity of the linear mappings in diagram (2.5), which we will use in the next section for the comparison of cohomology.

Lemma 2.1. In the diagram (2.5)

$$
\mu i=\pi_{*} \tilde{i}^{*},
$$

where $\mu$ is the degree of the mapping $\pi$.

REMARK. In other words, the diagram (2.5) is commutative up to a fixed constant multiple, which would not affect the passage to cohomology later on.

Proof. Outside of a proper analytic subset $\widetilde{S} \subset \tilde{X}$, the mapping $\pi$ is a finitely sheeted covering mapping of sheeting number $\mu$. Let $\pi(\widetilde{S})=S$, and thus we have that $\pi: \widetilde{X}-\widetilde{S} \rightarrow X-S$ is a locally biholomorphic covering mapping. By the Remmert proper mapping theorem $S$ is a proper analytic subset of $X$, and hence $S$ is of measure zero in $X$ (cf. Gunning and Rossi [9]). If $\phi \in \mathscr{E}^{p, q}(X)$, then the current $i(\varphi)$ is defined by its action on smooth forms with compact support, i.e., by

$$
\langle i(\varphi), \psi\rangle=\int_{X} \varphi \wedge \psi, \psi \in \mathscr{D}^{n-p, n-q}(X)
$$

Similarly, on $\tilde{X}$,

$$
\left\langle i\left(\pi^{*} \varphi\right), \tilde{\psi}\right\rangle=\int_{\tilde{X}} \varphi \wedge \widetilde{\psi}, \widetilde{\psi} \in \mathscr{D}^{n-p, n-q}(\tilde{X}),
$$

and $\pi_{*} i \pi^{*} \varphi$ is the restriction of the current $i \pi^{*} \varphi$ to $\pi^{*} \mathscr{D}^{n-p, n-q}(X) \subset$ $\mathscr{D}^{n-p, n-q}(\tilde{X})$. In other words,

$$
\begin{aligned}
\left\langle\pi_{*} i \pi^{*} \varphi, \psi\right\rangle & =\int_{\tilde{X}} \pi^{*} \varphi \wedge \pi^{*} \psi, \psi \in \mathscr{D}^{n-p, n-q}(X) \\
& =\int_{\tilde{X}} \pi^{*}(\varphi \wedge \psi)
\end{aligned}
$$


But we see that for $\varphi \in \mathscr{E}^{p, q}(X), \psi \in \mathscr{D}^{n-p, n-q}(X)$,

$$
\int_{\tilde{X}} \pi^{*}(\varphi \wedge \psi)=\int_{\tilde{X}-\tilde{s}} \pi^{*}(\varphi \wedge \psi)=\mu \int_{X-S} \varphi \wedge \psi=\mu \int_{X} \varphi \wedge \psi
$$

The middle equality in (2.9) follows easily by covering $X-S$ (in a locally finite manner) with open sets $\left\{U_{\alpha}\right\}$ so that $\pi^{-1}\left(U_{\alpha}\right)=U_{\alpha}^{1} U$ $\cdots \cup U_{\alpha}^{\mu}$, where $\left.\pi\right|_{U_{\alpha}^{j}}: U_{\alpha}^{j} \rightarrow U_{\alpha}$ is a biholomorphic mapping. Then letting $\left\{\rho_{\alpha}\right\}$ be a partition of unity for $\left\{U_{\alpha}\right\}$, we see that for $\eta \in \mathscr{E}^{n, n}(X)$

$$
\int_{\tilde{X}-\tilde{S}} \pi^{*} \eta=\sum_{\alpha} \int_{\pi^{-1}\left(U_{\alpha}\right)} \pi^{*} \rho_{\alpha} \eta=\sum_{\alpha} \mu \int_{U_{\alpha}} \rho_{\alpha} \eta=\mu \sum_{\alpha} \int_{U_{\alpha}} \rho_{\alpha} \eta=\mu \int_{X-S} \eta,
$$

since

$$
\int_{U_{\alpha}^{j}} \pi^{*} \rho_{\alpha} \eta=\int_{U_{\alpha}} \rho_{\alpha} \eta, \quad j=1, \cdots, \mu .
$$

Thus (2.6) follows easily from (2.7), (2.8), and (2.9).

We want to generalize the above results to differential forms with vector bundle coefficients. Suppose, as before $\pi: \tilde{X} \rightarrow X$ is a proper surjective mapping of complex manifolds of the same complex dimension $n$. Let $E \rightarrow X$ be a holomorphic vector bundle over $X$, and let $\widetilde{E}=\pi^{*} E$ be the pullback of $E$ by the mapping $\pi$. Letting $\mathscr{E}^{p, q}(X, E)$ be the differential forms on $X$ of type $(p, q)$ with coefficients in $E$, we have the diagram

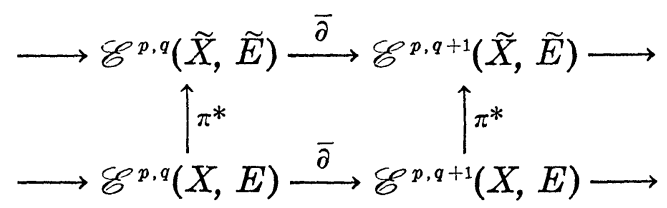

generalizing (2.1), where the $\bar{\partial}$ operator extends naturally to vectorvalued forms. Similarly, letting $E^{*}$ be the dual bundle to $E$, we have

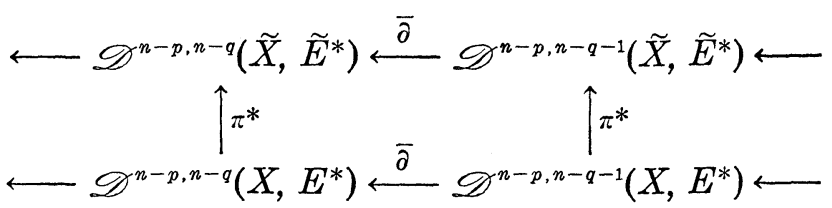

where $\widetilde{E}^{*}=\pi^{*} E^{*}$. By dualizing we obtain, in analogy to (2.5),

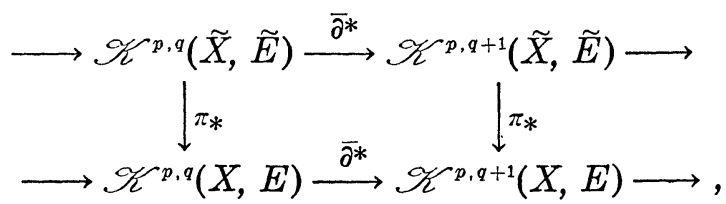


where $\mathscr{K}^{p, q}(X, E)$ is by definition the dual space to $\mathscr{D}^{n-p, n-q}\left(X, E^{*}\right)$. Moreover, $\bar{\partial}^{*}= \pm \bar{\partial}$ depending on the degree of the forms being acted on (cf. Serre [16]). Thus we have

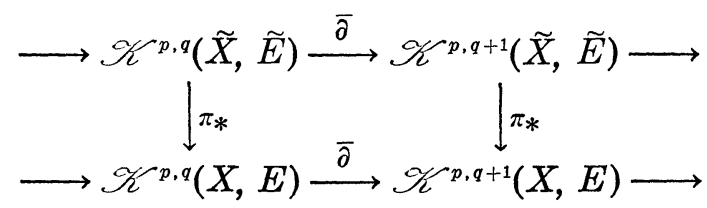

where each square is commutative or anticommutative. Note that locally, an element of $\mathscr{K}^{p, q}(X, E)$ can be written in the form

$$
\sum_{j=1}^{k} T_{j} \otimes e_{j}
$$

where $T_{j}$ is a current of type $(p, q)$, and $e_{j}$ is a holomorphic section of $E$, and the action of $\bar{\partial}$ is given by $\bar{\partial}\left(T_{j} \otimes e_{j}\right)=\bar{\partial} T_{j} \otimes e_{j}$, as in the case of $C^{\infty}$ forms. As before we consider the diagram

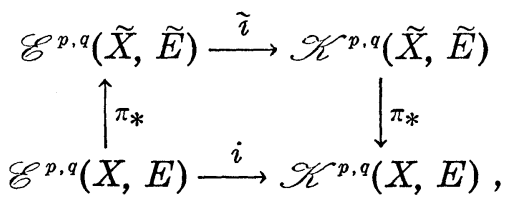

where $i$ and $\tilde{i}$ are the natural injections.

LeMma 2.2. If the mapping $\pi$ has degree $\mu$, then

$$
\mu i=\pi_{*} \tilde{i}^{*} \text {. }
$$

Proof. Suppose $\varphi \in \mathscr{E}^{p, q}(X, E)$ and $\psi \in \mathscr{E}^{n-p, n-q}\left(X, E^{*}\right)$, then one can give a meaning to and interpret $\varphi \wedge \psi$ as a scalar-valued differential form on $X$ of type $(n, n)$ (cf. Serre [7]). We do this locally, namely, if $\left(e_{1}, \cdots, e_{r}\right)$ is a local holomorphic frame for $E$ over an open set $U$ (i.e., $\left\{e_{1}, \cdots, e_{r}\right\}$ is a basis for $E_{x}$ for each point in $U$ ) and $\left(e_{1}^{*}, \cdots, e_{r}^{*}\right)$ is the dual frame for $E^{*}$, then we can express

$$
\begin{aligned}
& \varphi=\Sigma \varphi_{j} \otimes e_{j}, \varphi_{j} \in \mathscr{E}^{p, q}(U) \\
& \psi=\Sigma \psi_{j} \otimes e_{j}^{*}, \psi_{j} \in \mathscr{E}^{n-p, n-q}(U),
\end{aligned}
$$

and we let

$$
\varphi \wedge \psi=\Sigma \varphi_{j} \wedge \psi_{j} \in \mathscr{E}^{n, n}(U) .
$$

One can check that this definition of $\varphi \wedge \psi$ is independent of the local frame used, and does give a globally defined scalar-valued differential form. Using this we are able to interpret, for $\varphi \in$ $\mathscr{E}^{p, q}(X, E)$ 


$$
\langle i \varphi, \psi\rangle=\int_{X} \varphi \wedge \psi, \psi \in \mathscr{D}^{n-p, n-q}\left(X, E^{*}\right)
$$

Then the proof of this lemma follows in the same manner as the proof of Lemma 2.1.

Turning now to differentiable manifolds we want to prove the analogue of Lemmas 2.1 and 2.2. Suppose $\pi: \widetilde{Y} \rightarrow Y$ is a surjective proper differentiable mapping of orientable differentiable manifolds of the same dimension. Then we have the diagrams

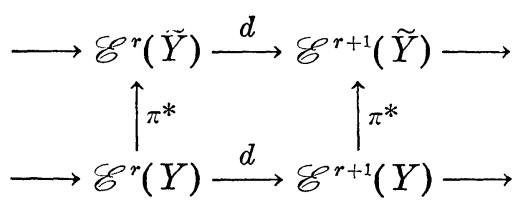

and

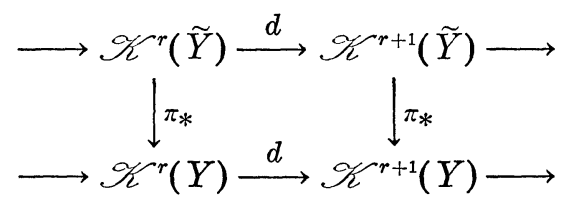

derived in the same manner as (2.1) and (2.4). Once again (2.15) is commutative, and (2.6) is commutative up to sign. Consider now the inclusion diagram

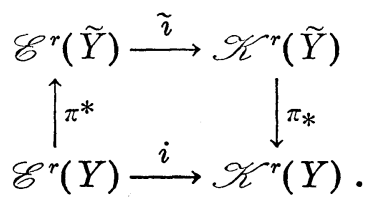

LEMMA 2.3. In the diagram (2.17)

$$
\mu i=\pi_{*} \tilde{i} \pi^{*}
$$

where $\mu$ is the degree of the mapping $\pi$.

Proof. We have to proceed somewhat differently in this case since the set of critical points $\widetilde{S}$ (points where $\pi$ has less than maximal rank) of $\pi$ is not necessarily of measure zero. Let $\pi(\widetilde{S})=$ $S$. Then it follows from Sard's lemma that $S$ has measure zero in $Y$ (cf. e.g., Sternberg [17], Chapter II). As in the proof of Lemma 2.1 , it suffices to show that for $\eta \in \mathscr{D}^{m}(Y)$, where $m=\operatorname{dim} Y$,

$$
\mu \int_{Y} \eta=\int_{\widetilde{Y}} \pi^{*} \eta
$$


But

$$
\int_{Y} \eta=\int_{\eta-S}
$$

Moreover, if $x \in \widetilde{S}, \eta \in \mathscr{D}^{m}(Y)$, then it follows that $\pi^{*} \eta(x)=0$. Namely in local coordinates $\left(y_{1}, \cdots, y_{m}\right)$ near $\pi(x)$, we have $\eta(y)=f(y) d y_{1} \wedge$ $\cdots \wedge d y_{m}$. Thus

$$
\pi^{*} \eta(x)=f(\pi(x)) d \pi_{1}(x) \wedge \cdots \wedge d \pi_{m}(x)=0,
$$

since $\pi$ is not maximal rank at $x \in \widetilde{S}$. Thus we have

$$
\int_{\tilde{Y}} \pi^{*} \eta=\int_{\tilde{Y}-\widetilde{S}} \pi^{*} \eta
$$

and we are reduced to showing that

$$
\mu \int_{Y-S} \eta=\int_{\tilde{Y}-\widetilde{S}} \pi^{*} \eta
$$

for the finitely sheeted covering mapping $\pi: \tilde{Y}-\widetilde{S}=Y-S$. This follows from the same arguments used in the proof of Lemma 2.1, but taking into account the orientations in the integrals over the local inverse images of a point $y_{0} \in Y$.

REMARK. We could have proved Lemma 2.1 in the same way using Sard's lemma instead of the fact that the set of points where a holomorphic mapping has less than maximal rank is a proper analytic subset.

3. Comparison of cohomology for ramified covering mappings. In this section we formulate and prove our results on injection of cohomology for ramified finitely-sheeted covering mappings, using the results from $\$ 2$.

THEOREM 3.1. Let $\pi: \widetilde{X} \rightarrow X$ be a proper surjective holomorphic mapping of complex manifolds of the same complex dimension, then $\pi$ induces injections for all $p, q$, and $r$ :

(a) $\pi^{*}: H^{q}\left(X, \Omega_{X}^{p}\right) \rightarrow H^{q}\left(\widetilde{X}, \Omega_{\widetilde{X}}^{p}\right)$,

(b) $\pi^{*}: H^{r}(X, C) \rightarrow H^{r}(\widetilde{X}, \boldsymbol{C})$,

(c) $\pi^{*}: H^{q}\left(X, \Omega_{X}^{p}(E)\right) \rightarrow H^{q}\left(\widetilde{X}, \Omega_{\widetilde{X}}^{p}\left(\pi^{*} E\right)\right)$, for any holomorphic vector bundle $E \rightarrow X$.

Let $X$ be a compact differentiable manifold, then let $b_{r}(X)=$ $\operatorname{dim}_{\boldsymbol{R}} H^{r}(X, \boldsymbol{R}), r=0, \cdots, \operatorname{dim} X$, be the Betti numbers of $X$. If $X$ is in addition a complex manifold we let $h^{p, q}(X)=\operatorname{dim}_{c} H^{q}\left(X, \Omega^{p}\right)$ be the 
Hodge numbers of $X^{2}$. Then we have the following corollary to Theorem 3.1.

Corollary 3.2. Let $\pi: \widetilde{X} \rightarrow X$ be a surjective holomorphic mapping of compact complex manifolds of the same dimension, then

$$
\begin{aligned}
b_{r}(X) & \leqq b_{r}(\widetilde{X}), \\
h^{p, q}(X) & \leqq h^{p, q}(\widetilde{X}) .
\end{aligned}
$$

More generally, we can generalize part (b) of the above Theorem 3.1 to the differentiable category. For simplicity we restrict our attention to orientable manifolds, since this avoids introducing differential forms of odd type (de Rham [5]). Analogous results can be obtained for unorientable manifolds by making suitable assumptions on the mapping $\pi$.

THEOREM 3.3. Let $\pi: \widetilde{X} \rightarrow X$ be a proper surjective mapping of orientable differentiable manifolds of the same dimension. Suppose $\mu=\operatorname{deg} \pi \neq 0$, then $\pi$ induces an injection on the de Rham groups:

$$
\pi^{*}: H^{r}(X, \boldsymbol{R}) \longrightarrow H^{r}(\tilde{X}, \boldsymbol{R}) \text {. }
$$

REMARK. (1) Such a result is definitely false for integral coefficients as the simple example of the covering mapping

$$
\pi: S^{2} \longrightarrow P_{2}(R)
$$

shows since $H^{1}\left(S^{2}, \boldsymbol{Z}\right)=0$, and $H^{1}\left(\boldsymbol{P}_{2}(\boldsymbol{R}), \boldsymbol{Z}\right)=\boldsymbol{Z}_{2}$.

(2) As mentioned in the introduction Hopf [10] proved that $\pi_{*}: H_{r}(\widetilde{X}, Z) /$ torsion $H_{r}(X, Z) /$ torsion is surjective (he assumed $\widetilde{X}$ is compact), which implies Theorem 3.1 in this case.

(3) Any mapping $\pi: S^{1} \rightarrow S^{1}$ of degree zero will not induce an injection $\pi^{*}: H^{1}\left(S^{1}, \boldsymbol{R}\right) \rightarrow H^{1}\left(S^{1}, \boldsymbol{R}\right)$.

In preparation for the proof of Theorems 3.1 and 3.2 we have to introduce various resolutions of the sheaves whose cohomology we are interested in. Let $X$ be a complex manifold, let $\mathscr{E}_{X}^{p, q}$ be the sheaf of germs of $C^{\infty}(p, q)$-forms on $X$, let $\mathscr{K}_{X}^{p, q}$ be the sheaf of germs of currents of type $(p, q)$, and let $\Omega_{X}^{p}$ be the sheaf of germs of holomorphic $p$-forms on $X$. It is well known that there are resolutions

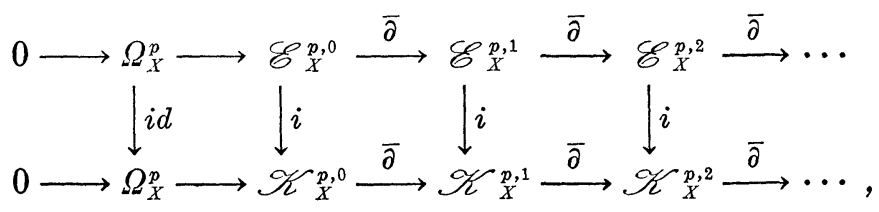

\footnotetext{
${ }^{2}$ Cf. e.g., Weil [18] or Wells [19].
} 
where the vertical maps $i$ in (3.1) are the natural injections of smooth $(p, q)$-forms into currents of type $(p, q)$ (see Serre [16]). Let

$$
\begin{aligned}
H_{\mathscr{E}}^{p . q} & =\frac{\operatorname{Ker}\left(\mathscr{E}_{X}^{p, q}(X) \stackrel{\bar{\partial}}{\longrightarrow} \mathscr{E}_{X}^{p, q+1}(X)\right)}{\operatorname{Im}\left(\mathscr{C}_{X}^{p, q-1}(X) \stackrel{\bar{\partial}}{\longrightarrow} \mathscr{C}_{X}^{p, q}(X)\right)} \\
H_{\mathscr{K}}^{p, q}(X) & =\frac{\operatorname{Ker}\left(\mathscr{K}_{X}^{p, q}(X) \stackrel{\bar{\partial}}{\longrightarrow} \mathscr{K}_{X}^{p, q+1}(X)\right)}{\operatorname{Im}\left(\mathscr{K}_{X}^{p, q}(X) \stackrel{\bar{\partial}}{\longrightarrow} \mathscr{K}_{X}^{p, q}(X)\right)}
\end{aligned}
$$

be the Dolbeault groups on $X$ with smooth and distribution coefficients, respectively. It follows that the injections $i$ in (3.1) induce an isomorphism

$$
H_{H^{q}(X, q}^{p, q}(X) \stackrel{i_{*}}{\longrightarrow} H_{\mathscr{Y}}^{p, q}(X),
$$

since the resolutions in (3.1) are fine resolutions.

Similarly if $X$ is a real differentiable manifold, we let $\mathscr{E}_{X}^{r}$ be the sheaf of real-valued differential forms of degree $r$, and let $\mathscr{K}_{X}^{r}$ be the sheaf of currents on $X$ of degree $r$. Then we have the (de Rham) resolutions

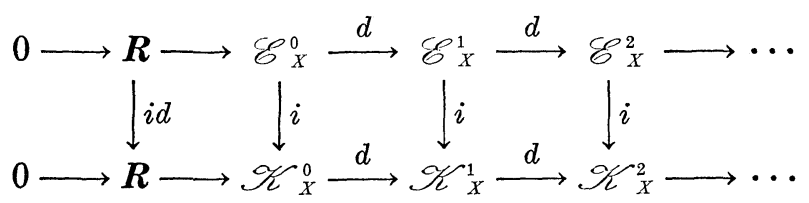

Consequently, if we let

$$
\begin{aligned}
& H_{\mathscr{E}}^{r}(X)=\frac{\operatorname{Ker}\left(\mathscr{E}^{r}(X) \stackrel{d}{\longrightarrow} \mathscr{E}^{r+1}(X)\right)}{\operatorname{Im}\left(\mathscr{E}^{r-1}(X) \stackrel{d}{\longrightarrow} \mathscr{E}^{r}(X)\right)} \\
& H_{\mathscr{K}}^{r}(X)=\frac{\operatorname{Ker}\left(\mathscr{K}^{r}(X) \stackrel{d}{\longrightarrow} \mathscr{K}^{r+1}(X)\right)}{\operatorname{Im}\left(\mathscr{K}^{r-1}(X) \stackrel{d}{\longrightarrow} \mathscr{K}^{r}(X)\right)},
\end{aligned}
$$

then we have the diagram

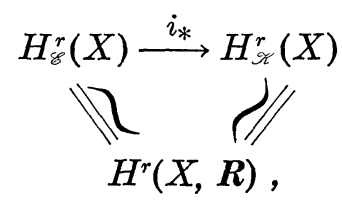

in analogy with (3.3), since (3.4) is a fine resolution of the constant 
sheaf $\boldsymbol{R}$. Note that the mappings $i$ in (3.1) and (3.4) are injections, whereas $i_{*}$ in (3.3) and (3.6) are isomorphisms at the cohomology level.

Proof of Theorem 3.1. We consider first part (a). Using (2.5) and (3.3) we obtain the diagram

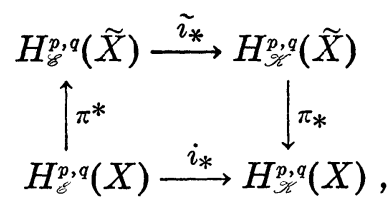

using the fact that $\pi^{*} \bar{\partial}=\bar{\partial} \pi^{*}$ and by duality $\pi_{*} \bar{\partial}= \pm \bar{\partial} \pi_{*}$, depending on the degree. By Lemma 2.1 we have that $\mu i=\pi_{*} \widetilde{i} \pi^{*}$, where $\mu$ is the degree of the mapping $\pi$. Moreover, $\widetilde{i}_{*}$ and $i_{*}$ are isomorphisms by (3.3). From this it follows immediately that $\pi^{*}$ is an injection. Namely, if $\pi^{*} \xi=0$, then $\pi_{*} \widetilde{i}_{*} \pi^{*} \xi=\mu i_{*} \xi=0$, and since $\mu \neq 0$, and $i_{*}$ is injective, it follows that $\xi=0$.

To prove (b) we proceed in exactly the same manner using Lemma 2.2 and (3.6), noting that $\pi^{*} d=d \pi^{*}$, and $d \pi_{*}= \pm \pi_{*} d$ as before.

To prove part (c) we tensor the resolutions (3.1) with $\mathcal{O}_{X}(E)$, the sheaf of holomorphic sections of the given holomorphic vector bundle, obtaining

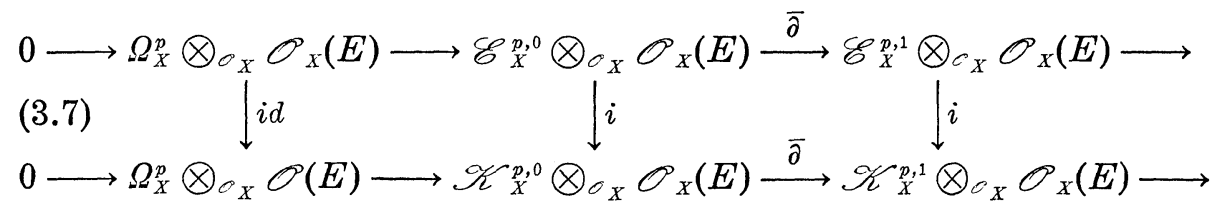

where we note that

$$
\Omega_{X}^{p}(E)=\mathscr{O}_{X}\left(\wedge^{p} T^{*}(X) \otimes E\right)=\Omega_{X}^{p} \otimes_{\mathcal{O}_{X}} \mathscr{\bigcirc}_{X}(E) .
$$

Thus (3.7) gives both $C^{\infty}$ and current resolutions of the sheaf $\Omega^{p}(E)$ of $E$-valued holomorphic $p$-forms. By writing down the $E$-valued analogue of (3.3) and using Lemma 2.2 as in the proof of part (a) above, part (c) follows immediately. We omit further details.

4. Kähler and symplectic manifolds. Let $Y$ be an even dimensional differentiable manifold of dimension $2 n$. If there is a differential form $\omega \in \mathscr{E}^{2}(Y)$ satisfying

(a) $d \omega=0$

(b) $\omega \wedge \cdots \wedge \omega \neq 0$ on $Y$

$n$ factors

then we say that $Y$ is a symplectic manifold and $\omega$ is called the symplectic form on $Y$. Note that a symplectic manifold is necessarily 
even dimensional (per definition) and orientable. For simplicity we let

$$
\begin{gathered}
\omega^{k}=\omega \wedge \cdots \wedge \omega, \\
k \text { factors }
\end{gathered}
$$

and thus $\omega^{n}$ is a volume element on $Y$. Suppose now that $X$ is a Kähler manifold with Kähler form $\omega$. Then the pair $(X, \omega)$ is a symplectic manifold (ignoring the complex structure). Recall that $\omega$ is a $d$-closed form of type $(1,1)$ on $X$ such that in local coordinates

$$
\omega=\frac{i}{2} \sum_{\alpha \beta} g_{\alpha \beta}(z) d z_{\alpha} d \bar{z}_{\beta}
$$

where the coefficient matrix $\left(g_{\alpha \beta}\right)$ is a Hermitian symmetric positive definite matrix. Moreover $\omega$ is real, i.e., $\omega=\bar{\omega}$, and it is the imaginary part of an Hermitian metric on $X$ (cf. [18], [19]).

We would like to generalize the results in $\S 3$ to proper surjective mappings of complex or differentiable manifolds where the fibres are no longer discrete, but are (generically) submanifolds of higher dimension. As we shall see by simple examples later on this is not always possible, but by restricting our attention to mappings of Kähler or symplectic manifolds, we get the same class of results.

THEOREM 4.1. Let $\pi: \widetilde{X} \rightarrow X$ be a proper surjective mapping of complex manifolds. Assume that $\tilde{X}$ is Kähler, then the following induced mappings on cohomology are injections:

(a) $\pi^{*}: H^{q}\left(X, \Omega_{X}^{p}\right) \rightarrow H^{q}\left(\tilde{X}, \Omega_{\widetilde{X}}^{p}\right)$,

(b) $\pi^{*}: H^{r}(X, C) \rightarrow H^{r}(\widetilde{X}, \boldsymbol{C})$,

(c) $\pi^{*}: H^{q}\left(X, \Omega_{X}^{p}(E)\right) \rightarrow H^{q}\left(\widetilde{X}, \Omega_{\tilde{X}}^{p}\left(\pi^{*} E\right)\right)$, for a holomorphic vector bundle $E \rightarrow X$.

Since every projective algebraic manifold is Kähler we have as a consequence.

Corollary 4.2. Let $\pi: \widetilde{X} \rightarrow X$ be a surjective holomorphic mapping of (compact) projective algebraic manifolds; then the conclusions of Theorem 4.1 hold, in particular

$$
\begin{aligned}
b_{r}(X) & \leqq b_{r}(\widetilde{X}), \\
h^{p, q}(X) & \leqq h^{p, q}(\widetilde{X}) .
\end{aligned}
$$

REMARK. At the end of this section we give an example of a proper surjective holomorphic mapping $\pi: \widetilde{X} \rightarrow X$ where $\tilde{X}$ is not Kähler and such that $\pi^{*}: H^{q}\left(X, \Omega_{X}^{p}\right) \rightarrow H^{q}\left(\widetilde{X}, \Omega_{\widetilde{X}}^{p}\right)$ is not injective. This shows that some hypothesis such as the Kähler assumption used 
above is necessary to get such a strong conclusion.

To prove these theorems we need an appropriate analogue of Lemma 2.1 in conjunction with the resolutions used before. However, note that in diagram (2.5) the mapping $\pi_{*}$ does not carry currents of type $(p, q)$ to currents of type $(p, q)$ if $\tilde{X}$ and $X$ do not have the same dimension. This is the problem when $\operatorname{dim} \tilde{X}>\operatorname{dim} X$, and it is at precisely this point that the Kähler (or symplectic) form will play a role. Since there is no difference in general between the Kähler and symplectic cases we will consider the Kähler case in detail first, and later on consider the modification necessary for the proof of Theorem 4.3.

Proof of Theorem 4.1. Suppose $\pi: \widetilde{X} \rightarrow X$ is a proper surjective holomorphic mapping and $\omega$ is a Kähler form on $\tilde{X}$. Suppose $\operatorname{dim}_{c} \tilde{X}=$ $m=n+d$, where $n=\operatorname{dim}_{c} X$, and suppose that $d>0$. Then it is easy to check that the induced mapping on currents (induced by duality from the mapping $\pi^{*}$ on forms) is of the form

$$
\pi_{*}: \mathscr{K}^{p+d, q+d}(\tilde{X}) \longrightarrow \mathscr{K}^{p, q}(X) \text {. }
$$

Moreover, $\pi_{*} \bar{\partial}= \pm \bar{\partial} \pi_{*}$, depending on degree. We want to define a mapping

$$
\tau: \mathscr{K}^{p, q}(\tilde{X}) \longrightarrow \mathscr{K}^{p, q}(X)
$$

to play the role of $\pi_{*}$ in (2.5). We first note that one can form the wedge product of a smooth form and a current, obtaining a new current. (cf. de Rham [5]). In particular, we can form the product $T \wedge \omega^{d}$, for $T \in \mathscr{K}^{p, q}(\widetilde{X})$, i.e., the action of $T \wedge \omega^{d}$ on forms with compact support is given by

$$
\left\langle T \wedge \omega^{d}, \dot{\psi}\right\rangle=\left\langle T, \omega^{d} \wedge \psi\right\rangle, \psi \in \mathscr{D}^{n-p, n-q}(X) .
$$

In particular $T \wedge \omega^{d} \in \mathscr{K}^{p+d, q+d}(\tilde{X})$. Thus we let

$$
\tau(T)=\pi_{*}\left(T \wedge \omega^{d}\right)
$$

and the mapping (4.1) is well defined. We now have the diagram

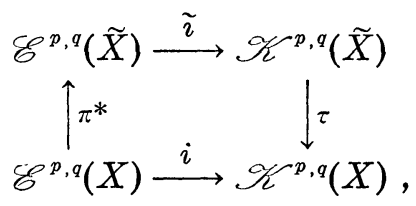

and the following lemma concerning the commutativity of (4.2).

Lemma 4.4. There exists a constant $\mu>0$ such that 


$$
\mu i=\tau \tilde{i} \pi^{*} .
$$

We will prove this lemma below. To complete the proof of Theorem 4.1(a) we note first that $\pi^{*}$ and $\tau$ commute (up to sign depending on the degree) with $\bar{\partial}$. Namely for $\tau$ we have

$$
\begin{aligned}
\bar{\partial}(\tau(T)) & =\bar{\partial}\left(\pi_{*}\left(T \wedge \omega^{d}\right)\right)= \pm \pi_{*}\left(\bar{\partial}\left(T \wedge \omega^{d}\right)\right) \\
& = \pm \pi_{*}\left(\bar{\partial} T \wedge \omega^{d}\right)= \pm \tau(\bar{\partial} T),
\end{aligned}
$$

since $\bar{\partial} \omega=\hat{\partial} \omega=0$. Thus we have the induced diagram on cohomology

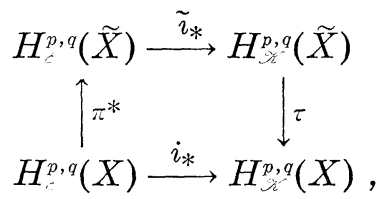

and by Lemma 4.4 we see that $\mu i_{*}=\pi * \tilde{i}_{*} \tau$. Thus it follows as before that $\pi^{*}$ is injective.

The proof of parts (b) and (c) are similar to the proof of parts (b) and (c) for Theorem 3.1, with the same modification used here given by the Kähler form and will be omitted.

Proof of Lemma 4.4. To prove Lemma 4.4 it suffices to show that there exists a constant $\mu>0$ so that

$$
\int_{\tilde{X}} \pi^{*}(\eta) \wedge \omega^{d}=\mu \int_{X} \eta,
$$

for any $\eta \in \mathscr{D}^{n, n}(X)$ (cf. the proof of Lemma 2.1). Once again we let $\widetilde{S}$ be the set of points where $\pi$ has less than maximal rank, and let $\pi(\widetilde{S})=S$. By using Sard's lemma again it suffices to show that (cf. the proof of Lemma 2.3)

$$
\int_{\tilde{X}-\tilde{S}} \pi^{*}(\eta) \wedge \omega^{d}=\mu \int_{X-S} \eta
$$

Now it is well known that $\pi: \widetilde{X}-\widetilde{S} \rightarrow X-S$ is a differentiable fibre bundle (cf. Kodaira-Spencer [13-III], Wells [19]), which is proven by introducing a Riemannian metric on $\widetilde{X}-\widetilde{S}$ and integrating an appropriate system of ordinary differential equations. Let $\tilde{Y}=$ $\widetilde{X}-\widetilde{S}$ and $Y=X-S$. Suppose for simplicity $\widetilde{Y}$ is a product manifold (locally it is since it is a fibre bundle), i.e., $\tilde{Y}=Y \times M$, and $\pi: Y \times$ $M \rightarrow Y$ is projection on the first factor, where $M$ is a compact differentiable manifold of real dimension $2 d$. Then we see that we have to compute

$$
\int_{Y \times M} \pi^{*} \eta(y) \wedge \omega^{d}(y, m)
$$


where we let $(y, m)$ denote the dependence on the two factors $Y \times$ $M$. Of course $\pi^{*}(\eta)(y)$ does not depend on the second factor. Then by Fubini's theorem we see that

$$
\int_{Y \times M} \pi^{*} \eta(y) \wedge \omega^{d}(y, m)=\int_{Y}\left[\int_{M} \omega^{d}(y, m)\right] \pi^{*} \eta(y) .
$$

Now in the general case when $Y$ is not necessarily a product we let $\left\{U_{\alpha}\right\}$ be a locally finite covering of $Y$ so that $\pi^{-1}\left(U_{\alpha}\right)$ is diffeomorphic to $U_{\alpha} \times M$, where $M=\pi^{-1}\left(y_{0}\right)$ for some fixed $y_{0} \in Y$. (We may assume without loss of generality that $Y$ is connected.) Let $\left\{\rho_{\alpha}\right\}$ be a partition of unity subordinate to $\left\{U_{\alpha}\right\}$, and then we may write

$$
\begin{gathered}
\int_{\widetilde{Y}} \pi^{*} \eta \wedge \omega^{d}=\sum_{\alpha} \int_{\pi^{-1}\left(U_{\alpha}\right)} \pi^{*} \rho_{\alpha} \eta \wedge \omega^{d} \\
=\sum_{\alpha} \int_{U_{\alpha}}\left[\int_{\pi^{-1}(y)} \omega^{d}\right] \rho_{\alpha}(y) \eta(y) \\
=\int_{Y}\left[\int_{\pi^{-1}(y)} \omega^{d}\right] \eta(y),
\end{gathered}
$$

where (4.4) follows as an application of (4.3), using the fibre preserving diffeomorphism $\pi^{-1}\left(U_{\alpha}\right) \cong U_{\alpha} \times M$. Thus the lemma will follow if we can show that

$$
\int_{\pi^{-1}(y)} \omega^{d}=f(y)
$$

is a constant positive function on $Y$. Clearly $f(y)>0$ for all $y$ since $f(y)$ is the volume of $\pi^{-1}(y)$ with respect to the volume element $\left.\omega^{d}\right|_{\pi^{-1}(y)}$. Now $\omega^{d}$ is a current of type $(d, d)$ on $\tilde{Y}$, and thus $\pi_{*} \omega^{d}$ is a current of type $(0,0)$ on $Y$, moreover since $d \pi_{*}=\pi_{*} d$, and $d \omega^{d}=$ 0 , we see that $d \pi_{*} \omega^{d}=0$, which implies by the regularity theorem for currents (cf. the resolution of $\boldsymbol{R}$ given by (3.4)) that $\pi_{*} \omega^{d}$ is a constant. We claim now that $\pi_{*} \omega^{d}$ is simply the function $f(y)$, gives by (4.6), considered as a current, and this will finish the proof of the lemma. If $\psi \in \mathscr{D}^{n, n}(Y)$, then

$$
\begin{aligned}
\left\langle\pi_{*} \omega^{d}, \psi\right\rangle & =\int_{\widetilde{Y}} \omega^{d} \wedge \pi^{*} \psi, \\
& =\int_{Y} f(y) \psi(y),
\end{aligned}
$$

as we saw above in (4.5) and therefore $\pi_{*} \omega^{d}$ as a current agrees with the function $f(y)$. Thus $f(y)$ must be constant.

We can generalize Theorem 4.1 to the differentiable category. 
Let $\pi: \widetilde{Y} \rightarrow Y$ be a proper surjective differentiable mapping of orientable even dimensional manifolds, where we assume that $\widetilde{Y}$ is symplectic with symplectic form $\omega$. Suppose that $2 d=\operatorname{dim} \tilde{Y}-\operatorname{dim} Y>$ 0 , and then consider $\mu=\pi_{*}\left(\omega^{d}\right) \in \mathscr{K}^{\circ}(Y)$. Then as before, $\mu$ will be constant on the components of $Y$, and we call $\mu$ the symplectic degree of the mapping $\pi$ (which depends on the choice of symplectic form $\omega$ ).

THEOREM 4.3. Let $\pi: \widetilde{Y} \rightarrow Y$ be a proper surjective differentiable mapping, where $\tilde{Y}$ and $Y$ are even dimensional orientable manifolds, and $\pi$ has nonzero symplectic degree. Then the induced mapping on de Rham cohomology

$$
\pi^{*}: H^{r}(Y, R) \longrightarrow H^{r}(\tilde{Y}, \boldsymbol{R})
$$

is an injection. In particular, if $Y$ and $\tilde{Y}$ are compact, then $b_{r}(Y) \leqq b_{r}(\tilde{Y})$.

The proof of Theorem 4.3 now follows in exactly the same manner as the proof of Theorem 4.1 using the symplectic form instead of the Kähler form. We omit further details.

REMARK. Theorem 4.3 is clearly false without an additional assumption (such as $\widetilde{Y}$ is symplectic) as the Hopf fibration $\pi: S^{3} \rightarrow S^{2}$ clearly does not induce an injection on de Rham cohomology.

We would now like to give an example of a proper surjective holomorphic mapping of complex manifolds $\pi: \widetilde{X} \rightarrow X$, where the conclusion of Theorem 4.1 is not valid. This will show that an additional assumption (such as $\tilde{X}$ being Kähler in Theorem 4.1) is necessary to conclude injection for the induced cohomology groups. Our example will be a Hopf surface $\widetilde{X}$ which is mapped surjectively onto $\boldsymbol{P}_{1}(\boldsymbol{C})$.

Explicitly, consider $C^{2}-\{0\}$, and let $\Gamma$ be the discrete group of automorphisms of $C^{2}-\{0\}$ defined by $\gamma\left(z_{1}, z_{2}\right)=\left(e^{m} z_{1}, e^{m} z_{2}\right), m \in Z$. Thus $\Gamma \cong Z$, and if we let $\widetilde{X}=\left(C^{2}-\{0\}\right) / \Gamma$ be the quotient space, and $\pi_{1}: C^{2}-\{0\} \rightarrow \tilde{X}$ be the quotient mapping, then $\tilde{X}$ is a compact complex manifold which is diffeomorphic to $S^{1} \times S^{3}$. This is one of the simplest examples of a compact complex manifold which is not Kähler (since $b_{1}(\tilde{X})=1$ ) and is due to Hopf (cf. [12], [19]). Consider the diagram

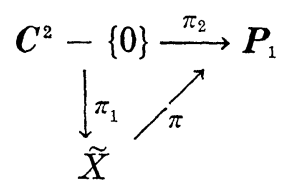


where $\pi_{1}$ is the usual projection of $C^{2}-\{0\}$ onto $\boldsymbol{P}_{1}\left(=\boldsymbol{P}_{1}(\boldsymbol{C})\right)$. Then the action of the group $\Gamma$ commutes with $\pi_{2}$ and thus $\pi_{2}$ induces the mapping $\pi: \widetilde{X} \rightarrow \boldsymbol{P}_{1}$. Now $h^{1,1}\left(\boldsymbol{P}_{1}\right)=1$, and we will have our desired example if we can show that $h^{1,1}(\tilde{X})=0$. This we will do now in several steps. First we will compute the other Hodge numbers for $\tilde{X}$ (following Kodaira [11]). We note first that $h^{2,0}(X) \leqq b_{2}(X)$ for any compact (complex) surface, and since $b_{2}(\tilde{X})=0$, we have $h^{2,0}(\tilde{X})$ $\left(=p_{g}(\tilde{X})\right.$, the geometric genus $)=0$. Thus $\tilde{X}$ is an elliptic surface in Kodaira's class $\mathrm{VII}_{0}$ (Kodaira [11]). Noether's formula for a compact surface is ${ }^{3}$

$$
12\left(h^{0,2}-h^{0,1}+1\right)=\int_{X} c_{1}^{2}+c_{2},
$$

where $c_{1}$ and $c_{2}$ are the Chern classes of $X$ (considered as differential forms in the de Rham group, for instance). Since $H^{2}(\widetilde{X}, C)=0$, we have $c_{1}^{2}=0$. Since $\widetilde{X} \cong S^{1} \times S^{3}$ it follows that $\int_{X} c_{2}=\chi(\widetilde{X})=0$ (the Euler characteristic). Consequently, we obtain that $h^{0,1}=1$. Then we have the Hodge numbers for $\widetilde{X}$ (using Serre duality)

$$
\begin{aligned}
& h^{2,0}=h^{0,2}=h^{1,0}=h^{1,2}=0 \\
& h^{0,1}=h^{2,1}=h^{0,0}=h^{2,2}=1 .
\end{aligned}
$$

We remark that any elliptic surface in Kodaira's class $\mathrm{VII}_{0}$ has the same Hodge numbers as in (4.7).

Fröhlicher proved in [7] that for any compact complex manifold $X$

$$
\sum_{p, q}(-1)^{p+q} h^{p, q}(X)=\chi(X) .
$$

Using the Hodge numbers from (4.7), along with the fact that the $\chi(\widetilde{X})=0$ vanishes, we obtain easily from $(4.8)$ that $h^{1,1}(\widetilde{X})=0$ (cf. Kodaira-Spencer [13-II]).

Thus, in summary, we have that

$$
\pi: \tilde{X} \longrightarrow P_{1}(C)
$$

is a surjective holomorphic mapping of compact complex manifolds which does not induce an injection on the induced mapping

$$
\pi^{*}: H^{1}\left(\boldsymbol{P}_{1}, \Omega_{\boldsymbol{P}_{1}}^{1}\right) \longrightarrow H^{1}\left(\tilde{X}, \Omega_{\widetilde{X}}^{1}\right),
$$

since $h^{1,1}(\tilde{X})=0$, and $h^{1,1}\left(\boldsymbol{P}_{1}\right)=1$.

REMARK. We want to mention one reason why the need for a

${ }^{3}$ Cf. Kodaira ]11]; this is a consequence of the Atiyah-Singer-Hirzebruch-RiemanRoch Theorem. 
Kähler metric disappears in Theorem 4.1 as soon as the fibre dimension becomes zero. Namely, the crucial ingredient in the proof of Theorem 4.1 is the fibre integral (4.6)

$$
f(y)=\int_{\pi^{-1}(y)} \omega^{d} .
$$

The function $f(y)$ is the volume of the fibre $\pi^{-1}(y)$ with respect to the metric $\omega$ given on $\tilde{X}$. The Kähler assumption $d \omega=0$ insures that the fibres all have the same volume. In the case of zero dimensional fibres, the volume $f(y)$ is merely the number of points in the inverse image (the degree of the mapping) which is also constant and independent of any metric on $\tilde{X}$.

\section{REFERENCES}

1. A. Aeppli, Modifikationen von reelen und komplexen Mannigfaltigkeiten, Comm. Math. Helv.. 31 (1956/7), 219-301.

2. A. Borel and A. Haefliger, La classe d'homologie fundamentale d'un espace analytique, Bull. Soc. Math. France, 89 (1961), 461-513.

3. T. Bloom and M. Herrera, De Rham cohomology of an analytic space, Invent. Math., 7 (1969), 275-296.

4. P. Deligne, Théorème de Lefschetz et critères de dégenéréscence de suites spectrales, Publ. Math. I.H.E.S., No. 35 (1968), 259-277.

5. G. de Rham, Variétés Differentiables, Hermann, Paris, 1955.

6. H. Federer, Geometric Measure Theory, Springer-Verlag, New York, 1969.

7. A. Fröhlicher, Relations between the cohomology groups of Dolbeault and topological invariants. Proc. Nat. Acad. Sci. U.S.A., 41 (1955), 641-644.

8. H. Grauert and O. Riemenschneider, Verschwindungssätze für analytische Kohomologie auf komplexen Räumen, Inv. Math., 11 (1970), 263-297.

9. R. C. Gunning and H. Rossi, Analytic Functions of Several Complex Variables, Prentice-Hall, Englewood Cliffs, N. J., 1965.

10. H. Hopf, Zur Algebra der Abbildungen von Mannigfaltigkeiten, J. Reine und Angewand. Math., 163 (1930), 71-88.

11. K. Kodaira, On the structure of compact complex analytic surfaces $I$, II, Amer. J. Math., 86 (1964), 751-798; 88 (1966), 682-721.

12. - Complex Structures on $S^{1} \times S^{3}$, Proc. Nat. Acad. Sci. U.S.A., 55 (1966), $240-243$.

13. K. Kodaira and D. C. Spencer, On deformations of complex analytic structures, I, II, Ann. of Math., 67 (1958), 328-466; III, Ann. of Math., 71 (1960), 43-76.

14. B. G. Moišezon, On n-dimensional compact varieties with $n$ aglebraically independent meromorphic functions, I, II, III, Amer. Math. Soc. Translat., 63 (2), (1967), 51-177, (Izvest. Akad. Nauk, SSSR, Ser. Mat., 30 133-174; 345-386, 621-656 (1966)).

15. O. Riemenschneider, Characterizing Moišezon spaces by almost coherent analytic sheaves, Math. Zeit., 123 (1971), 263-284.

16. J.-P. Serre, Un théorème de dualité, Comm. Math. Helv., 29 (1955), 9-25.

17. S. Sternberg, Lectures on Differential Geometry, Prentice-Hall, Englewood Cliffs, N. J., 1965.

18. A. Weil, Variétés Kähleriennes, Hermann, Paris, 1958.

19. R. O. Wells, Jr., Differential Analysis on Complex Manifolds, Prentice-Hall, Englewood Cliffs, N. J., 1973. 
R. O. WELLS, JR.

20. R. O. Wells, Jr., Moisezon spaces and the Kodaira embedding theorem, Proc. Conf. on Value Distribution Theory and Differential Geometry, Tulane University, (1973), (to appear).

Received June 25, 1973. Research supported by NSF Grant GP-19011 at Rice University and by the Universität Göttingen.

RICE UNIVERSITY 


\section{PACIFIC JOURNAL OF MATHEMATICS}

\section{EDITORS}

RICHARD ARENS (Managing Editor)

University of California

Los Angeles, California 90024

R. A. Beaumont

University of Washington

Seattle, Washington 98105
J. DugundJI

Department of Mathematics

University of Southern California

Los Angeles, California 90007

D. Gilbarg and J. Milgram

Stanford University

Stanford, California 94305

\section{ASSOCIATE EDITORS}

E. F. BECKENBACH

B. H. NeUMANN

F. WOLF

K. YosHIDA

\section{SUPPORTING INSTITUTIONS}

UNIVERSITY OF BRITISH COLUMBIA

CALIFORNIA INSTITUTE OF TECHNOLOGY

UNIVERSITY OF CALIFORNIA

MONTANA STATE UNIVERSITY

UNIVERSITY OF NEVADA

NEW MEXICO STATE UNIVERSITY

OREGON STATE UNIVERSITY

UNIVERSITY OF OREGON

OSAKA UNIVERSITY
UNIVERSITY OF SOUTHERN CALIFORNIA

STANFORD UNIVERSITY

UNIVERSITY OF TOKYO

UNIVERSITY OF UTAH

WASHINGTON STATE UNIVERSITY

UNIVERSITY OF WASHINGTON

$\stackrel{*}{*} \stackrel{*}{*} \stackrel{*}{*}$ AMERICAN MATHEMATICAL SOCIETY




\section{Pacific Journal of Mathematics}

\section{Vol. 53, No. $1 \quad$ March, 1974}

Martin Bartelt, Strongly unique best approximates to a function on a set, and a finite

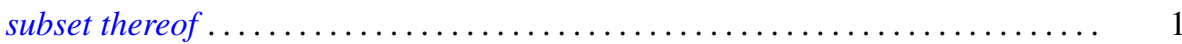

S. J. Bernau, Theorems of Korovkin type for $L_{p}$-spaces $\ldots \ldots \ldots \ldots \ldots \ldots \ldots \ldots \ldots$

S. J. Bernau and Howard E. Lacey, The range of a contractive projection on an

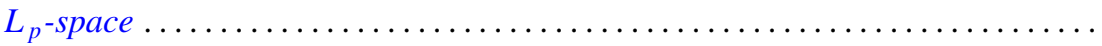

Marilyn Breen, Decomposition theorems for 3-convex subsets of the plane ......... Ronald Elroy Bruck, Jr., A common fixed point theorem for a commuting family of

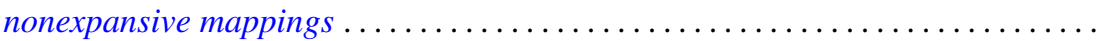

Aiden A. Bruen and J. C. Fisher, Blocking sets and complete $k$-arcs . . . . . . . 73

R. Creighton Buck, Approximation properties of vector valued functions . ......... 85

Mary Rodriguez Embry and Marvin Rosenblum, Spectra, tensor products, and

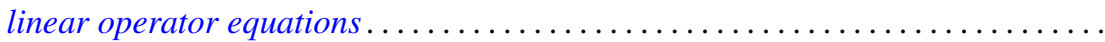

Edward William Formanek, Maximal quotient rings of group rings . . . . . . . . . 109

Barry J. Gardner, Some aspects of T-nilpotence . . . . . . . . . . . . . . . 117

Juan A. Gatica and William A. Kirk, A fixed point theorem for $k$-set-contractions

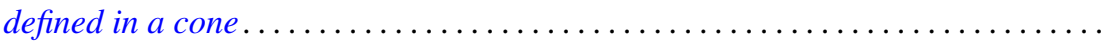

Kenneth R. Goodearl, Localization and splitting in hereditary noetherian prime

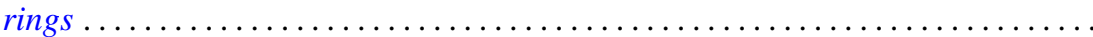

James Victor Herod, Generators for evolution systems with quasi continuous

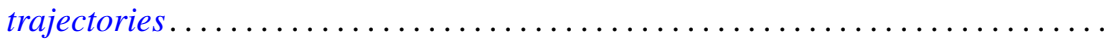

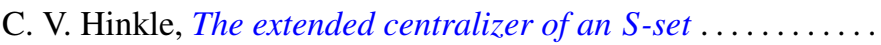

I. Martin (Irving) Isaacs, Lifting Brauer characters of p-solvable groups . . .

Bruce R. Johnson, Generalized Lerch zeta function ...........

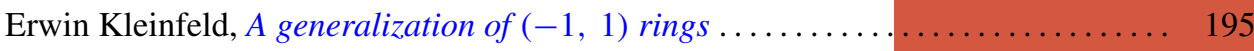

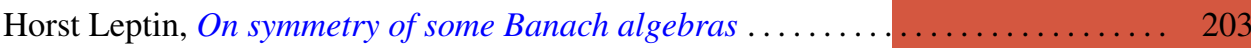

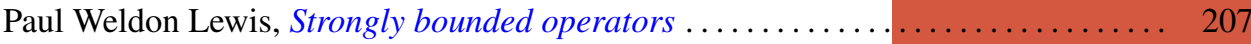

Arthur Larry Lieberman, Spectral distribution of the sum of self-adjoint

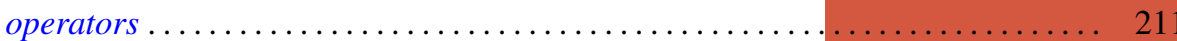

I. J. Maddox and Michael A. L. Willey, Continuous operators on paranormed spaces and matrix transformations

James Dolan Reid, On rings on groups ........................... 229

Richard Miles Schori and James Edward West, Hyperspaces of graphs are Hilbert cubes.

William H. Specht, A factorization theorem for p-constrained groups ...

Robert L Thele, Iterative techniques for approximation of fixed points of certain nonlinear mappings in Banach spaces ...............

Tim Eden Traynor, An elementary proof of the lifting theorem

Charles Irvin Vinsonhaler and William Jennings Wickless, Completely decomposable groups which admit only nilpotent multiplications .

Raymond O’Neil Wells, Jr, Comparison of de Rham and Dolbeault cohomology for

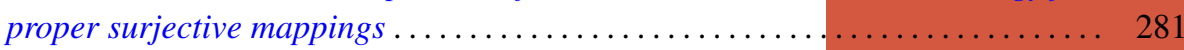

David Lee Wright, The non-minimality of induced central representations . . . . . 301 